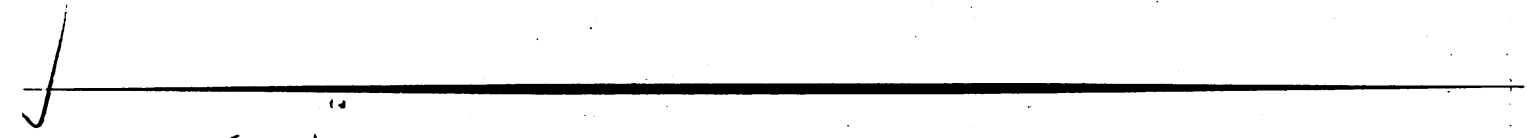

ROLE OF ALLERGY IN CLINICAL MEDICINE

By J. PEPYs, M.R.C.P.(LoND), M.R.C.P.(ED.)

PART I.

The assessment of the role of allergy in clinical medicine presents many difficult and confusing aspects. These must be clarified as far as possible if the scope of allergic methods of diagnosis and treatment is to be seen in its correct perspective, or indeed if they are to be applied at all. During the last half century the modern scientific concepts of anaphylaxis and allergy have been integrated into the theory and practice of medicine to a strikingly different degree in different countries and with marked fluctuations of interest in particular countries. The intimate relationship of clinical allergy to its source, the study of immunity, is evident in the early application of allergic methods to clinical medicine in countries where the study of immunity flourished, such as France, Germany, Austria and the U.S.A., and from which the greater part of the basic and and clinical studies of allergy have come. At present the U.S.A. and Scandinavian countries are making progress in immunity and there is at the same time a general acceptance of the role of allergy, which has become part of the general clinical teaching. In the British School of Medicine there have been many significant contributions to allergy and immunology during this period, but this has not been associated with any general integration into the body of medical thinking. In Britain the fluctuations of interest over the last 30 to 40 years have centred largely around the development of skin test techniques which promised a direct etiological approach to the group of difficult clinical allergic disorders. The frequent failure to realize this promise, for reasons which will be discussed and of which the absence of an adequate understanding of the immunological mechanisms is important, has resulted in an illogical dissociation of the theoretical and experimental aspects of allergy from their significance in relation to clinical allergic disorders. 'Allergy' is therefore commonly included in lists of differential diagnoses and the mechanisms may be referred to, without any attempt to test its principles by practical application. This too-ready dismissal has led physicians to overlook extensive accumulated clinical experience and to an inadequate analysis of the climical problems. This is evident daily in the histories and progress of patients to whom allergic methods are carefully and conscientiously applied, and it is proposed here to analyze some of the questions which are regularly brought forward as reasons for failure or reluctance to draw a direct association between basic allergic mechanisms and clinical disorders.

\section{History of Clinical Allergy}

Recorded observations of clinical 'allergic conditions preceded the explanation of the mechanisms involved and the formulation of the concept of ' allergy' by a considerable period. There are records dating from antiquity of a causal relationship between exposure to innocuous substances and the production of minor or severe clinical disorders. Systematic observations were carried out in the century before the origin of the modern concept of allergy, that is from 1800-1900, which provided support for this possibility. In 1800 , Robert Bree, of London, in a dissertation on asthma, described as one of the causes the exposure to hair powder, which he observed causing sneezing and asthma in certain subjects. In 1819, Bostock, of Guy's Hospital, described ' hay fever' as a clinical entity, and in 1831 Elliotson, of the University of London, pointed out the relationship of hay fever to grass and flower pollen. Many studies of the mechanism of allergic reactions have been carried out by physicians on themselves and Blackley, of Manchester, in investigations from $1856-1873$ proved on himself the causal relationship of pollen to hay fever. He elicited skin test reactions by applying pollen to his skin, and nasal reactions by applying it to his nasal mucosa. Most significantly he found that nasal tests with pollen outside the hay fever season 
elicited typical hay fever symptoms and he described, too, a causal relationship between exposure to animal dander and the inhalation of mould spores and the production of asthmatic reactions. He was a pioneer of the study of pollen concentration in the air and carried out pollen counts on exposed glass slides. $\mathrm{He}$ was able to correlate these counts with the incidence of hay fever symptoms and he also reported the presence of mould spores on his slides, a finding of topical interest in view of the recent emphasis on allergic reactions to mould spores. Wyman, of Harvard ( 1872$)$, described the hereditary tendency in hay fever and suggested that other plants, dust, animal emanations and foods could also provoke symptoms. In 1859, Hyde-Salter, of Charing Cross Hospital, reported in a discussion of asthma that 40 per cent. of his patients gave a family history of asthma, and that he had observed closely the causal relationship between exposure to animal emanations and food and the production of asthma. He obtained positive reactions to skin tests on himself with cat dander, exposure to which gave him asthma. Amongst the examples he cited was a child who developed asthma on ingesting milk, and medical students who developed asthma on exposure to ipecacuanha powder. Provocative factors which were commonly recognized included fog, changes of temperature, winds, heavy meals and exertion, and interestingly enough Hyde-Salter pointed out the undesirability of treatment of asthma with opium.

In spite of these studies and of Blackley's careful and readily reproducible observations on the effect of pollen in hay fever, other views were also held at that time. Beard in 1876 claimed that ' hay fever is essentially a neurosis.' It is curious to find that in 1874 a hay fever association was formed in the U.S.A., which had as an article of its constitution that, 'it is the duty of each member to report to the recording secretary the discovery of any remedy, source of relief, or exempt district which may come to his or her knowledge during their natural life; and afterward, if permitted.' A little reflection will indicate that the conflicting views have not yet been resolved, perhaps because none of the members of the hay fever association has yet been permitted to report back to the recording secretary.

The formulation of the modern scientific concept of allergy by Richet, v. Pirquet and others provided the explanation for the previously observed clinical phenomena. With the passage of 40 to 50 years the correlation of the experimental and clinical findings has been confirmed repeatedly, and techniques of primary importance at one period have assumed a different role at another.
This applies in particular to skin testing and will be discussed.

\section{Scope of Allergy in Clinical Medicine}

The more recent history and present status of $\stackrel{\overrightarrow{\vec{D}}}{\rightarrow}$ clinical allergy are reflected in the types of textbooks in current use. These indicate the wide $\overline{ }$ and specialised forms which it is taking, and $\frac{\bar{\sigma}}{\widehat{D}}$ reflect the systemic nature of allergic disorders $\stackrel{\mathbb{Q}}{\Omega}$ with their recognized occurrence in almost all के parts and systems of the body. Comprehensive general texts have been produced by:

HANSEL, F. K. (r936), 'Allergy of the Nose and Paranasal Sinuses.'

VAUGHAN, W. T. (r939), 'Practice of Allergy.'

COOKE, R. A. (1947), 'Allergy in Theory and 워 Practice.'

FEINBERG, S. M. (1946), 'Allergy in Practice.' ఝ్

URBACH, E., and GOTTLIEB, P. (1946), $\underset{V}{\mathcal{V}}$ 'Allergy.'

SULZBERGER, M. B. (1940), ' Dermatological 을 Allergy.'

SCHWARTZ, L., TULIPAN, L., and PECK, $\bigcirc$ S. M. (1947), 'Occupational Diseases of the $\frac{0}{0}$ Skin.'

RINKEL, H. J., RUDOLPH, T. G., ang $\frac{\vec{\theta}}{\theta}$ ZELLER, M. ('95I), ' Food Allergy.' 㝏

ABRAHAMSON, H. A. (I95I), ' Treatment Asthma.'

CHOBOT, R. (195I), ' Paediatric Allergy.' ' SHELDON, J. M., LOVELL, R. G., and MATTHEWS, K. P. (1953), 'Manual of $\frac{\varrho}{8}$ Clinical Allergy.'

Most of these volumes are the product of $\overrightarrow{\vec{A}}$ combined contributions from specialists in most 3 fields of medicine and illustrate the pervading influence of allergic disorders in general medicine. This makes it desirable for the physician treating an allergic disorder in a special field to be able to 3 . recognize, assess, and perhaps treat manifestations in other systems, since these frequently co-exist. The physician should be aware of and recognize $\delta$ allergic factors and the allergist in turn should recognize non-allergic factors. The latter require- 윽 ment has been expressed by Vaughan, who adapted the clinical aphorism that "to know syphilis is to know medicine' by saying that 'to know allergy one must know medicine.'

The search for improved techniques of in- $\tilde{O}$ vestigation and treatment of allergic disorders $\mathrm{N}$ continues, but until it becomes possible to controlo the constitutional tendencies which permit these $\varphi$ conditions to develop and manifest themselves, the application of existing methods provides the ? only practical approach. In order to do this $T$ adequately the following appear to be prime requirements : 
(I) An understanding of the concepts involved in the basic mechanisms of allergic reactions. This serves to explain the rationale for the measures adopted and the different stages in the evolution of allergic reactions at which modifying influences can exert an effect. The primary initiating role of allergic mechanisms is made clearer and the experimental evidence to support this has been discussed in this journal (Pepys, 1953).

(2) The relationship between these mechanisms and clinical disorders should be considered in regard to:

(a) The social significance of allergic disorders in the population as a whole, and possible methods of decreasing their incidence.

(b) The treatment and care of the individual subject.

\section{Social Significance of Allergic Disorders}

The high incidence of allergic disorders is commonly recognized by practitioners in most branches of medicine. Factual evidence supports this and indicates the magnitude of their influence on the life of the individual and on society. The data to be given here are taken from an analysis of the social importance of allergic diseases by Dr. D. A. Williams of Cardiff (r95I). He in turn has based his survey on the Registrar General's report ànd on other public health statistical reports. The prevalence of major allergic disorders for which treatment is demanded among the general population is generally accepted to be ro per cent. in the U.S.A. and Finland, and a similar figure is found in Britain. The incidence of active allergic illnesses in relation to all other diseases is as follows: asthma, I/I IO; hay tever, I/I00; migraine, I/400; urticaria, I/3,800; and allergic eczema, I/I I,000. Allergic disorders are slightly less prevalent than all forms of arthritis or high blood pressure; are commoner than influenza, peptic ulcer or all forms of anaemia, and are three times commoner than tuberculosis. The importance of asthma among the allergic disorders is indicated by the opinion of the American Academy of Allergy that, if asthmatics were excluded, the problem of allergy would be of no great importance in the Armed Forces. The effect of asthma on life is indicated by the fact that asthma deaths are 0.6 per cent. of total deaths from all causes and that the average per annum for the years $1938-48$ was 2,915 . The maximum incidence of asthma deaths is in the age group of 60 to 64 years, and the lowest between 5 to 14 years. In the population as a whole the incidence of asthma was $9 / 1,000$, whereas university students of Wales and Cambridge gave a history of asthma in $46 / 1,000$ and of active asthma in $21 / 1,000$. Asthma is seventh on the list of defined diseases as a cause of incapacity, producing $206 / 10,000$ due to all causes, with migraine producing 23/10,000. Asthma itself produces: twice the incapacity of pulmonary tuberculosis; more than gastric and duodenal ulcer; more than all forms of arthritis; and ten times that of rheumatic heart disease. Asthma is three times as common as pulmonary tuberculosis or diabetes or thyroid conditions, five times as common as rheumatic disease and six times as common as pernicious anaemia. Out of 3,000,000 hospital discharges in England and Wales, allergic diseases accounted for 147,000 and asthma accounted for II 1,000 of these, thus indicating the burden on hospital facilities. Demands on the doctor are also heavy and allergic disorders require more consultations than all forms of anaemia; almost as many as gastric, duodenal and anastomotic ulcers combined and as all forms of arthritis, and asthma itself is eighth on the list of disorders requiring medical attention.

Vaughan (I939) found a history of minor allergic episodes, due to occasional contacts, in 50 per cent. of the population, and major allergic episodes, due to additional commoner contacts, in ro per cent., the difference between the two groups depending largely on the degree and frequency of contact with the responsible factors.

Implicit in these findings is the need for $(a)$ the provision of facilities for the practice of allergy as a part of clinical medicine and for organize training in this subject and (b) the fullest application of existing knowledge to decrease the incidence and severity of allergic disorders in the population as a whole. The discussion of allergic and other factors in relation to the individual subject will indicate the points at which prevention can be applied effectively.

Features of Clinical Allergy in the Individual

The similarity between experimental studies and clinical allergy and the possibility of making direct deductions from experimental studies has been pointed out (Pepys, 1953). For those who are not familiar with the theoretical or experimental basis of allergy or who have not witnessed, for example, the high degree of hypersensitivity which frequently exists, and the exquisite specificity of antigen which may be required to elicit a reaction, there are several concepts which can make this association clearer and provide clear-cut examples on which to base discussion.

The definition of 'allergy' by v. Pirquet postulated a previous adequate exposure to the allergen in order to produce a state of specific hypersensitivity, which may be termed the ' allergic state.' The subject can remain entirely unaware of this unless there is adequate re- 
exposure to the specific allergen in the future, perhaps for the duration of life, or unless the dose of antigen is so large that it persists in the body sufficiently long to provoke a reaction when the allergic state is produced, as for example in serum sickness. Apparently unrelated substances may contain chemically related allergenic fractions which should be considered in cases where a direct association is not obvious. The duration of exposure needed to produce the allergic state may vary from patient to patient and depends on the degree and circumstances of the exposure. Dutton (1938) found that occupational exposure to sugar beet pollens for two seasons was required in order to produce hypersensitivity in an appreciable number of workers.

Salen and Juhlin-Dannfelt (1935) found a high percentage of positive reactions on testing following occupational exposures, thus 38 per cent. of 125 bakers gave positive reactions to a rye extract in comparison with only 5 per cent. of the controls, and 23 per cent. of Ioo cavalrymen gave positive reactions to horse dander as compared with 8 per cent. in allergic patients. The study of hay fever in Japanese subjects by Hara (1935, 1939) provides interesting evidence along these lines, since it was found that the absence of hay fever in Japan is associated with the almost entire absence of grass pollen on slides exposed in Japanese cities, in spite of the presence of grasses known to be able to cause hay fever in the islands. Japanese living in Calitornia, however, had an incidence of hay fever of 3.5 per cent. compared with other races, and they required 5 to I 5 years to acquire hay fever. Comparison of ragweed pollen hay fever in American-born persons and those coming to America from Europe where ragweed is absent showed that the European-born subject acquired hay fever later in life, but required the same average incubation period regardless of the age of first exposure (Clarke and Leopold, 1940). Equally clear instances are seen in laboratory workers who may take $\mathrm{I} \frac{1}{2}$ to 2 years' exposure to become hypersensitive to animal danders. With certain drugs hypersensitivity may develop very early and after very small dosage, and aspirin allergy is a good example of this (Prickman and Buchstein, 1937; Pepys, 195I).

The importance of the need for exposure to an allergen, in order to produce hypersensitivity towards it, cannot be stressed too much. This fundamental aspect, which is abundantly proven by experimental evidence, provides a means of applying preventive measures. In the absence of adequate exposure, hypersensitivity towards a specific allergen does not occur. The route of exposure influences the ease of sensitization and needs to be kept in mind in the use of therapeutic sub- ̊ stances.

Hereditary Factor in the Production of the $\frac{\varrho}{.}$ Allergic State

An hereditary influence in allergic conditions $\stackrel{\frac{\rho}{+}}{\rightarrow}$ has long been reported, but whilst it is generally accepted that it is the tendency to develop hyper- $\frac{\bar{\sigma}}{\bar{\omega}}$ sensitivity or the ' allergic state' which is inherited, $\vec{\odot}$ there are other points of view which must be dis- $\varrho$ cussed. Coca (1923) devised the term 'atopy' to is describe allergic subjects, with a family history of $\vec{\circ}$ allergy, with a tendency to develop spontaneous or natural hypersensitivity and possessing what were $\vec{\omega}$ thought to be distinctive immunological charac- $\frac{\Omega}{0}$ teristics. The apparent differences between allergic disorders in this group and those in whom hypersensitivity is produced by artificial means, 0 such as by injection or ingestion of drugs, were $\omega$ discussed (Pepys, 1953), and are no longer valid. The distinction between ' natural' and 'arti- $-T$ ficial' exposure is clearly unsatisfactory. The sole remaining feature of Coca's concept is the ease of sensitization of the ' atopic' group, which $\overrightarrow{0}$ is strikingly influenced by the family history of allergy, so that commonly encountered sub- $\frac{0}{0}$ stances readily produce hypersensitivity, which is $\stackrel{\mathbb{C}}{-}$ frequently multiple. The description of the hypersensitivity as 'natural' unfortunately give the impression of inevitability and of a poer prospect for prevention. There are other tacto which may permit sensitization to occur without being recognized, thus giving the semblance of spontaneity to a clinical reaction occurring later.

There is no general agreement on intra-uterine $\varrho$ sensitization as postulated by Ratner, Jackson and $\overrightarrow{\vec{O}}$ Gruehl (1927), though the production of $\mathrm{Rh}-3$ incompatibility indicates that this is possible. A likely source of sensitization is through the breast milk and Donnally (1930) has reported that egg white can be demonstrated immunologically in the breast milk of women after ingestion of egg, and he quotes a number of other reports in which. claims are made that allergic reactions in intants $O$ can be produced by feeding, or relieved by with- $₹$ holding foods from the mother. In this way an 음 infant can become sensitized and may manifest $>$ hypersensitivity before ingesting a particular food directly. Allergic reactions to mother's milk may N well be due to such a process. The excretion of ${ }^{\circ}$ drugs in the mother's milk is well known and 0 supports this possibility.

The hereditary influence may act in two ways: (a) by influencing the tendency to develop hyper-o sensitivity and (b) by determining the "shock $\Phi$ organ,' i.e. the type of clinical disorder. In a $\stackrel{\oplus}{+}$ recent study of heredity in bronchial asthma, $\square$ Schwartz (1952) takes the view that it is the asthma 
which is interited and that the development of hypersensitivity is secondary. He claims that the significance of exposure to allergens such as baker's flour for example, is to give an occupational character to the disorder. There are certain allergic disorders in which the heredity factory influences strongly the nature of the clinical disorder, such as migraine, and angioneurotic oedema affecting the larynx (Sheldon, I949). The view that the tendency to develop hypersensitivity is the more important is nevertheless generally accepted, and further analysis may be helpful. The predominance of asthma among the allergic disorders can be explained in several ways, which make Schwartz's views less tenable. In the majority of patients with allergic conditions of the respiratory tract, immediate type hypersensitivity is present, so that contact with the allergen results in an immediate exudative reaction together with smooth muscle contraction. The influence of the distribution of smooth muscle on species differences in the clinical picture of anaphylaxis has been discussed (Pepys, 1953), and in man the large amount of smooth muscle in the lungs makes it a common 'shock organ,' this being enhanced in turn by the fact that inhalant allergens are the commonest causes of respiratory tract allergy, so that the antigen antibody reaction takes place at the portal of entry, that is in the respiratory tract. The development of systemic hypersensitivity in these cases is seen from the skin and conjunctival reactions which occur on contact with the allergen, and not infrequently these reactions may precede the development of asthma. The evidence favours the inheritance of an inherited abnormality or liability of an immunological nature. This enables the recognition of the population most at risk, for whom control of environmental exposure can be of preventive value. The report by Pappenheimer and Kuhns (1952), on the production of ' incomplete' antibodies in allergic subjects after diphtheria immunization, may clarify this aspect further, since these antibodies are skin-sensitizing, and testing with the antigen produced immediate type whealing reactions, in the same way as with antibodies produced in response to ordinary allergens. The possibility that allergic subjects inherit the tendency to produce a particular type of antibody merits much further study.
The incidence of allergic disorders in families of allergic subjects is shown by Schwartz's figures. He found that, if one parent had asthma, 50 per cent. of the children will carry the gene, and 40 per cent. of them, that is 20 per cent. of all the children, will develop manifest disease; in 13 per cent. of them asthma, and in the remaining 7 per cent. vasomotor rhinitis, Besnieres prurigo or hay fever. If both parents are asthmatic, 75 to 100 per cent. of the children will carry the gene and 30 to 40 per cent. will manifest disease, with asthma in 20 to 25 per cent. In females of healthy parents trom allergic stock, it is impossible to predict whether they will carry the gene, and at 25 to 30 years more than half will have manifested disease. If earlier children have allergic manifestations, the incidence in siblings will be higher, and 60 to 100 per cent. of the siblings of asthmatic parents will develop disease.

In an early paper, Spain and Cooke (1924) reported that over $5 \circ$ per cent. of allergic subjects have a history of allergy in their antecedents. With a bilateral family history of allergy, 75 per cent. of the children develop allergic disorders, whereas with a unilateral history $5 \circ$ per cent. do so. A negative family history was found in 40 per cent. of cases. The age of onset was younger in children with a bilateral family history, 36 per cent. presenting symptoms in the first five years, whereas with a unilateral inheritance the maximum in cidence was between ro to 15 years, and with a negative family history the maximum incidence was at 30 years.

The two salient facts which arise out of the discussion so far are:

(I) Adequate exposure to the allergen is required before specific hypersensitivity, i.e. the ' allergic state,' is established. 'The adequate exposure is determined in part by the degree and frequency of contact with potential allergens, this making commoner environmental factors predominant among the allergenic factors.

(2) In addition to the factor of exposure to the allergen, the tendency to become sensitized torms the other aspect. The population most at risk can be determined from the family history, since the hereditary tendency is very potent. Both factors can therefore be readily established, namely the commoner potential allergens, and the individuals most likely to be sensitized. 